\title{
Experimental and Numerical Study on the Permeation Grouting Diffusion Mechanism considering Filtration Effects
}

\author{
Guangxuan Zhu, ${ }^{1}$ Qingsong Zhang $\mathbb{D}^{1}{ }^{1}$ Rentai Liu, ${ }^{1}$ Jiwen Bai, ${ }^{1}$ Wei Li, ${ }^{1}$ and Xiao Feng ${ }^{2}$ \\ ${ }^{1}$ Geotechnical and Structural Engineering Research Center, Shandong University, Jinan 250061, China \\ ${ }^{2}$ School of Transportation Engineering, Shandong Jianzhu University, Jinan 250101, China \\ Correspondence should be addressed to Qingsong Zhang; zhangqingsong@sdu.edu.cn
}

Received 7 November 2020; Revised 17 December 2020; Accepted 4 January 2021; Published 15 January 2021

Academic Editor: Bin Gong

Copyright ( 2021 Guangxuan Zhu et al. This is an open access article distributed under the Creative Commons Attribution License, which permits unrestricted use, distribution, and reproduction in any medium, provided the original work is properly cited.

\begin{abstract}
Permeation grouting estimation is important for the design of grouting engineering. Filtration effects and rheological behavior play a key role in permeation grouting diffusion of cement-based grouts. To better understand the effect of filtration and grout rheological behavior on the grouting diffusion mechanism, one-dimensional permeation grout injections in sand columns under constant flow rate were performed by a self-developed experimental procedure. Experimental results showed that there were dramatic variations in rheological parameters and porosity along the diffusion distance. However, the rheological parameters changed slightly with time for each position. Based on the experimental results, a numerical model considering the filtration effect and grout rheological behavior was established to describe the mechanism of grout flow in porous media. In addition, numerical solutions from the proposed model are compared with the experimental results. The comparative results showed that the proposed numerical method can match the laboratory tests well. Finally, the effects of the grout flow velocity and the water/cement ratio of the grout on the diffusion mechanism are also discussed.
\end{abstract}

\section{Introduction}

Subway tunnels are usually shallow and often pass through water-rich sand layers. In the construction of subway tunnels, water leakage treatment has become a great challenge. Water leakage will cause the loss of sand particles in the ground and induce the disaster of water and sand inrush; thus, threatening the long-term safety of aboveground buildings and underground pipelines. In addition, water leakage will accelerate the corrosion of equipment and may compromise driving safety. The typical water leakage in subway tunnels is shown in Figure 1.

Permeation grouting with cement-based grout has become an effective method for water leakage treatment because of its convenient construction and friendliness to the environment. Permeation grouting is an effective method used for enhancing stabilization and reducing the permeability of granular soil deposits without disturbing the original soil structure. In the past decades, the permeation grouting technique has been widely used in various civil engineering applications [1-4]. The grouting diffusion radius is critical to the design of the permeation grouting projects. Generally, the grouting diffusion pressure reflected indirectly the permeation diffusion processes $[5,6]$. However, it is difficult to predict the grouting diffusion distance, because of the complexity and concealment of permeation grouting.

Generally, chemical grout has a low viscosity and its gel time can be adjusted accurately. Moreover, there is no filtration effect in the process of grouting diffusion. Although chemical grouts have better injectability, cement-based suspensions remain the most widely used materials in permeation grouting projects on the basis of lower costs, higher durability, and/or better environmental safety [3, 7]. Filtration has an important effect on the diffusion of the cementbased grout. It occurs when cement particles are filtered from the grout by the soil skeleton. The filtered cement particles become part of the soil skeleton and block the grout diffusion channel, thus decreasing the porosity and permeability of the soil. In addition, grout concentration decreases continuously. These behaviors greatly influence the variation in the grouting pressure. Therefore, understanding the filtration mechanism is of great importance for the design of permeation 


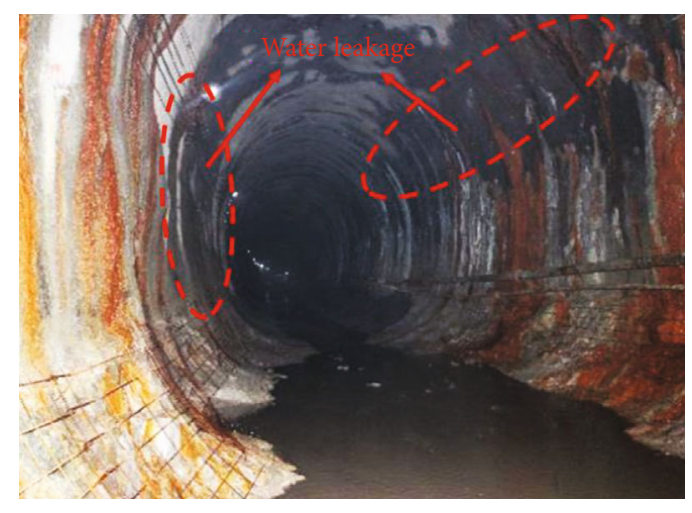

Figure 1: Water leakage in the subway tunnel.

grouting engineering. The mechanism of filtration has not been studied in detail in grouting applications.

To date, lots of experimental researches have been done to study the filtration effects of suspended particles in porous media. The majority of experiments have investigated filtration effects based on the variation of grouting pressure and effluent particle concentration through the porous media, using a common cylinder column [8-11]. Furthermore, the lengths of the cylinder column varied. Ahfir et al. [12] investigated the effect of the column length and the pore flow velocity on the suspended particle deposition. Moreover, several factors have been found to influence the filtration mechanism. For instance, the particle size distribution [13, 14], flow rate, porous media grain size, and particle chemical properties have a significant impact on filtration behavior. The filtration coefficient can be calculated using the mass of the grout injected and the injection pressure $[15,16]$. In the above research, the columns were treated as an integral part. The spatial and temporal variation of porosity and concentration of grout were ignored along the column length. In contrast, some visualization techniques have been developed in experiments to study the filtration effect in porous media. For instance, the process of particle suspension transport within porous media was directly observed at the pore scale by an epi-fluorescent microscope and other devices [17]. Xray tomography and nuclear magnetic resonance (NMR) imaging techniques are suitable for scanning the threedimensional structures of porous media or obtaining the velocity field of the slurry. However, it is still difficult to obtain the variation of the slurry and porous media [1821]. Above all, the variation of porosity, permeability, and grout concentration in porous media has not been revealed in most of these experiments. The temporal and spatial variation of slurry and the porosity in porous media are very important for understanding the permeation grouting diffusion mechanism.

In addition to experimental studies, numerical studies have been conducted to investigate the filtration phenomenon in porous media. For instance, a typical theoretic model was proposed by Reddi et al. [22] to study the filtration behavior in permeation grouting. Subsequently, based on a one-dimensional Darcy flow, Kim et al. [23] gave closedform solutions using a stepwise numerical calculation. In addition, some studies indicate that very low variations of the porosity may induce high variations of permeability $[16,24,25]$. A theoretical model of cement suspension flow in granular porous media considering particle filtration was presented by Saada et al. [16], based on a phenomenological law predicting high variations of permeability for low variations of porosity. In most theoretic models, a first-order rate law is used to describe the filtration effects. A key parameter in the continuum model is the deposition coefficient. After extensive parametric studies, Kim and Whittle [26] draw a conclusion that the first-order rate law is not able to describe the filtration effects accurately and a more complex model is necessary. However, the measurement of the parameters in a complex model is difficult. A reliable method is needed to determine the model parameters for the modelling of the filtration effect.

In this research, one-dimensional permeation grout injections in sand columns under constant flow rate were carried out. The temporal and spatial variation of the grout rheological characteristics and the porosity of porous media were revealed. Moreover, the rheological characteristics of the grout front during injection were analyzed. To describe the penetration diffusion of cement-based grout in the sand, a numerical model was developed, which considered the filtration effect and the grout rheological characteristics. Numerical solutions of the proposed theoretical model were able to match the laboratory tests well. In addition, the effect of the water/cement ratio and the grouting flow rate on the diffusion mechanism was studied.

\section{Experimental Test}

In this section, the one-dimensional permeation grouting experiments under constant flow rates are described. The rheological properties of cement-based grout with different water-cement ratios were measured. The relationship between the rheological parameters and the concentration of grout was obtained by fitting. The temporal and spatial variations of sand porosity and grout rheological properties during permeation grouting were analyzed. The experimental results reveal the influence of the filtration effect on the permeation grouting diffusion mechanism.

\subsection{Materials}

2.1.1. Cement. The cement used in this study was an ordinary Portland cement (PO.42.5) with a fineness of $330-410 \mathrm{~m}^{2} / \mathrm{kg}$ and a specific gravity of $3.14 \mathrm{~g} / \mathrm{cm}^{3}$. The cement particles with a size of over $80 \mu \mathrm{m}$ were removed using the negative pressure sieving method. Cement grout with water-cement ratios (WCR) of 1.0, 1.5, and 2.0 were prepared. To obtain a stable cement slurry, a blender (Q1U-FF07-160) and a churn barrel (300 $\mathrm{mm}$ in diameter and $450 \mathrm{~mm}$ in height) were used. In the churn barrel, the cement-based grout was mixed for a total of $10 \mathrm{~min}$ in three stages: $4 \mathrm{~min}$ at $400 \mathrm{r} / \mathrm{min}, 2 \mathrm{~min}$ at $300 \mathrm{r} / \mathrm{min}$, and finally $4 \mathrm{~min}$ at $400 \mathrm{r} / \mathrm{min}$. The components of the cement are listed in Table 1.

To obtain its rheological characteristics, the cement grout was tested using seven water/cement ratios. After mixing, 
TABLE 1: The chemical composition of cement in the test.

\begin{tabular}{lcccccc}
\hline Ignition loss & $\mathrm{SiO}_{2}$ & $\mathrm{Fe}_{2} \mathrm{O}_{3}$ & $\mathrm{Al}_{2} \mathrm{O}_{3}$ & $\mathrm{CaO}$ & $\mathrm{MgO}$ & $\mathrm{SO}_{3}$ \\
\hline $0.56 \%$ & $19.45 \%$ & $4.42 \%$ & $5.84 \%$ & $63.31 \%$ & $4.38 \%$ & $2.6 \%$ \\
\hline
\end{tabular}

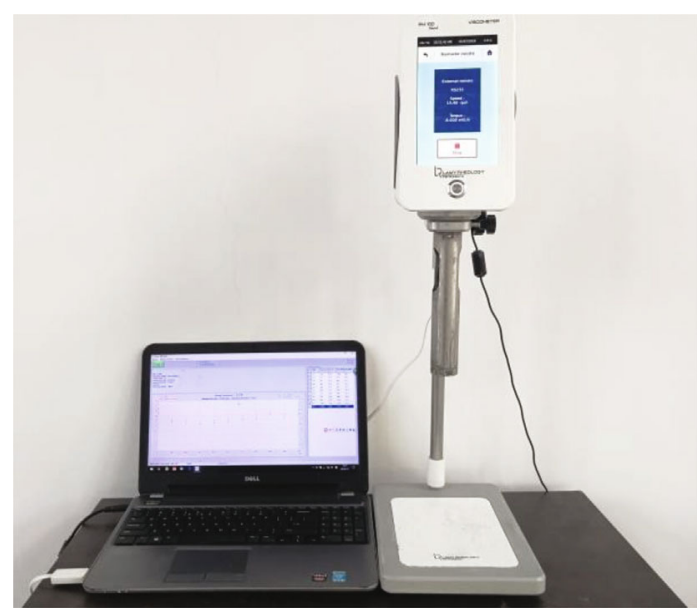

FIgURe 2: Lamy RM100 touch-type rheometer.

$20 \mathrm{~mL}$ of the grout sample was placed into a beaker vessel for rheology tests. Grout rheology tests were conducted at room temperature $\left(21 \pm 1^{\circ} \mathrm{C}\right)$ using an RM100 touch-type rheometer equipped with a coaxial cylinder spindle. All measurements were carried out at $t_{0}$ ( $30 \mathrm{~s}$ after mixing). The measurements were carried out by varying progressively the shear rate from 100 to $1000 \mathrm{~s}^{-1}$. The measured shear stress values were taken after a minimum of $30 \mathrm{~s}$ without fluctuation. The yield stress $\tau_{0}$ and the viscosity of the slurry $\mu_{0}$ were determined for each sample by fitting a Bingham model $\left(\tau=\tau_{0}+\mu \gamma\right)$ to the linear descending portion of the flow model. The Lamy RM100 touch-type rheometer and the rheology results are shown in Figure 2. The rheological properties and the determined coefficients are presented in Table 2.

The concentration of cement grout $c$ can be related to the water/cement ratio of the grout [26] as follows:

$$
c=\frac{1}{1+\left(\rho_{s} / \rho_{w}\right) \cdot(W / C)}
$$

where $\rho_{\mathrm{w}}$ and $\rho_{\mathrm{s}}$ are the density of water and solid particles, respectively, and $W$ and $C$ are the mass of water and cement, respectively.

For the yield stress and viscosity of the grout, two nonlinear regressions of the points, according to the concentration of cement grout, were performed:

$$
\begin{aligned}
\tau_{0} & =4.57 \times 10^{-6} \times e^{47.84 c}, \\
\mu & =\mu_{w}+0.1279 c-0.2631 c^{2},
\end{aligned}
$$

where $\mu$ and $\mu_{\mathrm{w}}$ are the viscosity of grout and water, respectively.

2.1.2. Sand. The sand was obtained from Qingdao, China. The sand in the experiments was prepared by a vibratory
TABLE 2: Measured rheological properties and density of the tested grouts.

\begin{tabular}{lcc}
\hline WCR & Yield strength $\tau_{0} / \mathrm{Pa}$ & Viscosity $\mu / \mathrm{mPa} \cdot \mathrm{s}$ \\
\hline 1.0 & 0.8593 & 16.9 \\
1.1 & 0.3266 & 16.4 \\
1.2 & 0.1136 & 15.9 \\
1.5 & 0.0559 & 15.6 \\
2.0 & 0.0357 & 14.2 \\
5.0 & 0.0121 & 8.5 \\
10.0 & 0.0057 & 4.6 \\
\hline
\end{tabular}

sieve shaker and six standard sieves of $0.05,0.15,0.2$, $0.4,0.6$, and $1.2 \mathrm{~mm}$. The specific gravity of the sand grains was measured by a gas pycnometer (ASTMD555000). The parameters of the sand samples used in the experiment are presented in Table 3 . Figure 3 shows the sand grain size distribution curve determined by dry sieving (ASTMD421\&D422).

2.2. Experiment Equipment. The self-developed onedimensional permeation grouting diffusion test device is shown in Figure 4. This device consists of a grout supply system and a diffusion system. The grout supply system includes a steel grout container, a hydraulic cylinder, a hydraulic pressure station, and a computer with a servo control system. The steel grout container has a volume of $60 \mathrm{~L}$. The maximum pressure output by the grout supply system is approximately 1.5 $\mathrm{MPa}$. The device is suitable for both constant pressure and constant flow experiments.

The hydraulic pressure station can be controlled by the computer via the servo control system. The hydraulic pressure station has a pressure sensor, which can monitor the cylinder oil pressure in real time. The oil pressure can be further converted into grouting pressure. The grouting flow rate and the pressure in the barrel can be controlled accurately by the computer. Meanwhile, the grouting pressure can be measured automatically during the constant grouting flow rate test.

The diffusion system includes a cylinder vessel and six grout collectors. The cylinder vessel consists of six sections with an internal diameter of $100 \mathrm{~mm}$ and a length of $1800 \mathrm{~mm}$. Each section of the cylinder was bolted with the adjacent parts. A small hole was drilled into each section of the cylinder at a specific location to serve as a slurry outlet. The grout in the cylinder vessel flows into the grout collector through the hole controlled by the outlet valves. The spacing of adjacent holes is $300 \mathrm{~mm}$.

2.3. Experimental Procedures. These experiments were carried out at room temperature. Before each experiment, fiber fabrics were placed at both ends of the steel-walled cylinder to avoid any clogging of the ducts by soil particles. A given quantity of the sand was placed into the steel cylinder and statically compacted to a porosity of 0.4 . To ensure the uniformity of the specimen in the filling process, the sand specimen was filled with layers of $100 \mathrm{~mm}$ in thickness. 
TABLE 3: Parameters of the grouted medium.

\begin{tabular}{lccccccc}
\hline & \multicolumn{4}{c}{ Particle size proportion (\%) } & & \multirow{2}{*}{ Bulk density $\left(\mathrm{kg} / \mathrm{m}^{3}\right)$} & \multirow{2}{*}{ Particle density $\left(\mathrm{kg} / \mathrm{m}^{3}\right)$} \\
\hline $1.05 \mathrm{~mm}$ & $0.15 \mathrm{~mm}$ & $0.2 \mathrm{~mm}$ & $0.4 \mathrm{~mm}$ & $0.6 \mathrm{~mm}$ & $1.2 \mathrm{~mm}$ & 1690 & 2630 \\
\hline
\end{tabular}

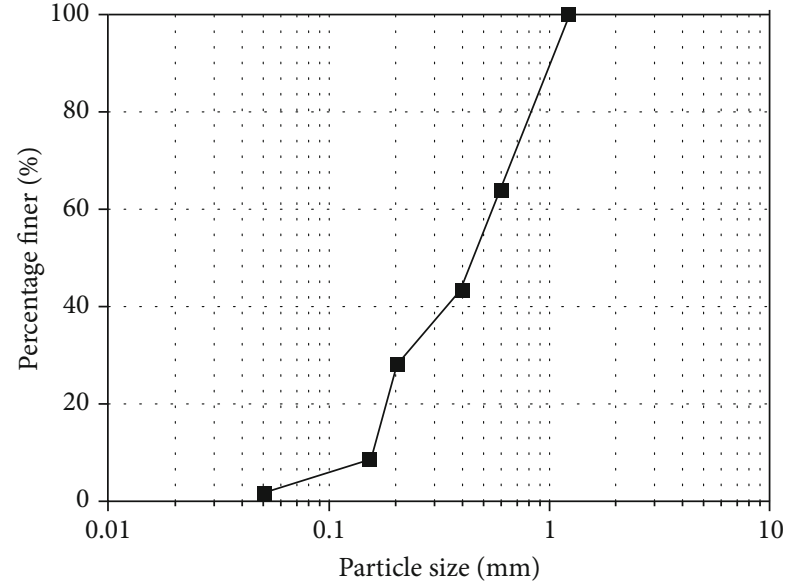

Figure 3: Grain size distribution curves of the test sand.

Before each grouting experiment, water was injected into the sand column until the flow rate of water was steady, thereby removing the air in the sand voids. Subsequently, cement grout was produced according to the design of the water/cement ratio. Then, the cement grout was poured into the grout container, and the outlet valves on the steel cylinder were opened in order for the penetration grouting with a constant flow rate to begin. As soon as the grout flowed to the outlet valve, the grout was collected. The amount of grout collected was $20 \mathrm{~mL}$ every 20 seconds. The rheological parameters of the grout collected were measured immediately after each experiment using the RM100 touch-type rheometer. After each experiment, the steel cylinders with the grouted sand sample were left for 28 days. Subsequently, the solidified sand samples were taken out from the steel cylinder and machined into cylinder samples with $30 \mathrm{~mm}$ in diameter and $30 \mathrm{~mm}$ in height. The porosity and permeability of the grouted sand samples were measured.

To study the evolution of sand porosity and the rheological parameters of grout (i.e., viscosity and yield stress), cement grouts were injected into the same sand sample at different grouting times ranging from 20 to $140 \mathrm{~s}$ (no.1-no.7). The effect of grouting flow rate was investigated by injecting the grout with a flow rate of $1.45 \mathrm{~L} / \mathrm{min}$ (no.8) and $4.30 \mathrm{~L} / \mathrm{min}$ (no.9) at the same water/cement ratio of 1.5. To assess the effect of the water/cement ratio, two injections were performed at the same grouting flow rate of $2.85 \mathrm{~L} / \mathrm{min}$ with different water/cement ratios: 1 (no.10) and 2 (no.11). The details of the tests conducted are listed in Table 4.

2.4. Experimental Results. The temporal and spatial variation of cement slurry viscosity is shown in Figure 5(a). As shown in the figure, the viscosity at any location has a slight increase with time. In test no.7, the grouting flow rate was $2.85 \mathrm{~L} / \mathrm{min}$ and the porosity of the sand sample was 0.4 . It can be calculated that the diffusion velocity of the grout front was $15 \mathrm{~mm} / \mathrm{s}$. Therefore, the dotted line represents the change of viscosity of the grout diffusion front during the injection. Because of the filtration effect, cement particles in the grout diffusion front were filtered. With the injection, the concentration and viscosity of the grout diffusion front decreased continuously. Furthermore, the attenuation rate of the grout viscosity decreased continuously along the direction of grouting diffusion.

The temporal and spatial variations of the cement grout yield stress are shown in Figure 5(b). As shown in the figure, the viscosity at any location changed slightly with time. However, along the direction of the grout diffusion, the yield stress decreased significantly. The yield stresses of cement gout on the diffusion front at $0,0.3,0.6$, and $0.9 \mathrm{~m}$ were $24,1.6,0.2$, and $0.05 \mathrm{mPa} \cdot \mathrm{s}$, respectively. The above phenomena demonstrate that the filtration effect occurred during the injection.

The spatial and temporal variation of porosity in the sand column is shown in Figure 6(a). As shown in the figure, the porosity attenuation decreases with the increase of grouting diffusion distance. At the inlet $(0 \mathrm{~m})$ of the sand column, the time for grout deposition was the longest and the amount of cement particles deposited was the largest. Therefore, the attenuation of porosity in the sand column at the column inlet was the largest, and the porosity of the sand column was the smallest. At the time of $140 \mathrm{~s}$, the porosity of the sand column at the inlet was 0.19 , and the attenuation ratio was $52.5 \%$. However, at any position of the sand column, the porosity attenuation rate changed slightly with time. At the inlet, the porosity attenuation rate was approximately $1.6 \times 10^{-3} \mathrm{~s}^{-1}$.

The spatial and temporal variation of permeability in the sand column is shown in Figure 6(b). As shown in the figure, the permeability of the sand column varied nonlinearly along the direction of grouting diffusion. Combining the data shown in Figures 6(a) and 6(b) for comprehensive analysis, the permeability decreased sharply with the porosity reduced from 0.4 to 0.36 . Then, the porosity further decreased from 0.36 , and the permeability reduced slightly. At the inlet, the initial permeability of the sand column was $4.0 \times 10^{-9} \mathrm{~m}^{2}$. Compared with the initial permeability at the inlet, the attenuation rate of permeability in the sand column at 20,40, and 60 s was $87.5 \%, 93.75 \%$, and $96.9 \%$, respectively, while the porosity attenuation rate remained at approximately $1.5 \times$ $10^{-3} \mathrm{~s}^{-1}$. The experimental results show that very low variations of porosity may induce high permeability variations at the beginning of grouting. As a matter of fact, porosity is a macroparameter of porous media. In porous media, only part of the space among the sand particles are effective channels for grout flow [27]. Other parts of the space among the sand particles do not contribute to particle migration. Therefore, the deposition of cement particles in the flow channel 


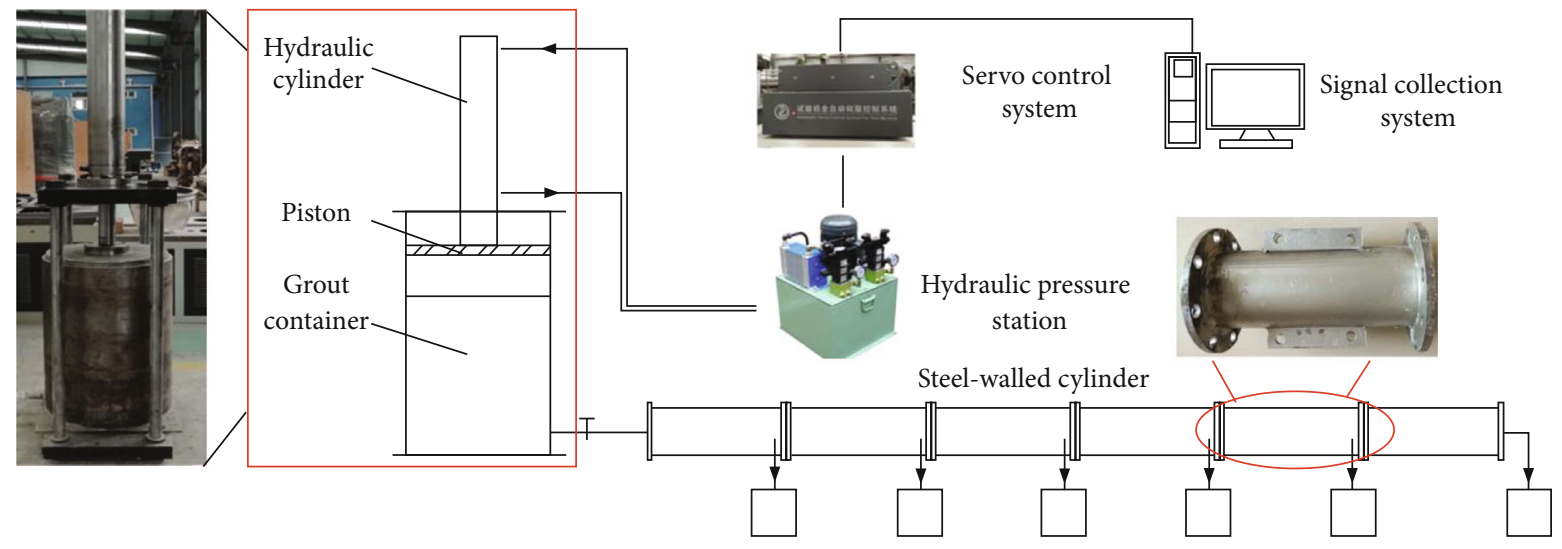

FIGURE 4: Schematic diagram of the test system.

TABle 4: Experimental designs.

\begin{tabular}{lccc}
\hline No. & Grouting flow rate & Water/cement ratio & Terminal time/s \\
\hline 1 & $2.85 \mathrm{~L} / \mathrm{min}$ & 1.5 & 20 \\
2 & $2.85 \mathrm{~L} / \mathrm{min}$ & 1.5 & 40 \\
3 & $2.85 \mathrm{~L} / \mathrm{min}$ & 1.5 & 60 \\
4 & $2.85 \mathrm{~L} / \mathrm{min}$ & 1.5 & 80 \\
5 & $2.85 \mathrm{~L} / \mathrm{min}$ & 1.5 & 100 \\
6 & $2.85 \mathrm{~L} / \mathrm{min}$ & 1.5 & 120 \\
7 & $2.85 \mathrm{~L} / \mathrm{min}$ & 1.5 & 140 \\
8 & $1.45 \mathrm{~L} / \mathrm{min}$ & 1.5 & 120 \\
9 & $4.30 \mathrm{~L} / \mathrm{min}$ & 1.5 & 40 \\
10 & $2.85 \mathrm{~L} / \mathrm{min}$ & 1 & 60 \\
11 & $2.85 \mathrm{~L} / \mathrm{min}$ & 2 & 60 \\
\hline
\end{tabular}

changes the permeability of the porous media more prominently compared to the change in porosity.

Uniform permeation grout diffusion in the sand is critical to the study of the filtration effect. In this study, the grout diffusion distances were measured. The volume of grout filling sand voids shall be equal to the total volume of the slurry injected. According to the mass balance of grout, the grout diffusion distance can be calculated at any time in the condition of a constant flow rate. The measurements and the calculations of diffusion distance are presented in Figure 7. There is good agreement between the measurements and the calculations. Uniform permeation grouting was proven.

In this section, the temporal and spatial variation of the grout rheological parameters, sand porosity, and grouted sand permeability were revealed. This contributes to understanding the diffusion mechanism of permeation grouting. However, how the water-cement ratio of cement grout and grouting flow rate affect the grouting diffusion mechanism, respectively? And how the grouting diffusion distance can be determined according to the grouting pressure?

\section{Numerical Model Describing Grout Flow with Filtration Behavior}

Based on the experimental results, a numerical model considering the filtration effect and the grout rheological behavior was established to describe the diffusion mechanism of grout in porous media. A method is proposed to determine these coefficients.

3.1. Basic Assumptions. Before presenting the model, the assumptions that were made are described:

(1) Cement grout can be regarded as a Bingham fluid [28], thus being incompressible. From Table 2, the yield stress of cement grout cannot be ignored, especially for the water-cement ratio varied between 1 and 2 . The yield stress and the viscosity of cement grout varied depending on the water/cement ratios. A Bingham fluid constitutive model can be described well

(2) With injection, the deformation of the sand skeleton could be ignored. The sand matrix is regarded as rigid $[16,29]$

(3) The porous medium is homogeneous and isotropic

(4) The equivalent homogeneous cylinder channel was used to describe the pores in the porous media [22]

(5) Under the condition of constant rate grouting, the slurry diffusion conforms to laminar flow

3.2. Governing Equations. The seepage diffusion in porous media can be described by a homogeneous capillary model. According to the Poiseuille equation, the total flow in a circular tube is expressed as follows.

For a Bingham fluid, the flow $\bar{v}$ through a single tube can be predicted by:

$$
\bar{v}=\frac{b^{2}}{8} \frac{1}{\mu}\left[-\frac{d p}{d l}-\frac{8}{3} \frac{\tau_{0}}{b}-\frac{1}{3}\left(\frac{2 \tau_{0}}{b}\right)^{4}\left(\frac{d p}{d l}\right)^{-3}\right],
$$

where $\bar{v}$ is the average flow velocity in the single seepage pipeline, $b$ is the radius of the seepage pipeline, $\tau_{0}$ is the yield stress of the slurry, $2 \tau_{0} / b=\zeta$ is the slurry starting pressure gradient, $p$ is the seepage pressure, and $l$ is the grout diffusion 


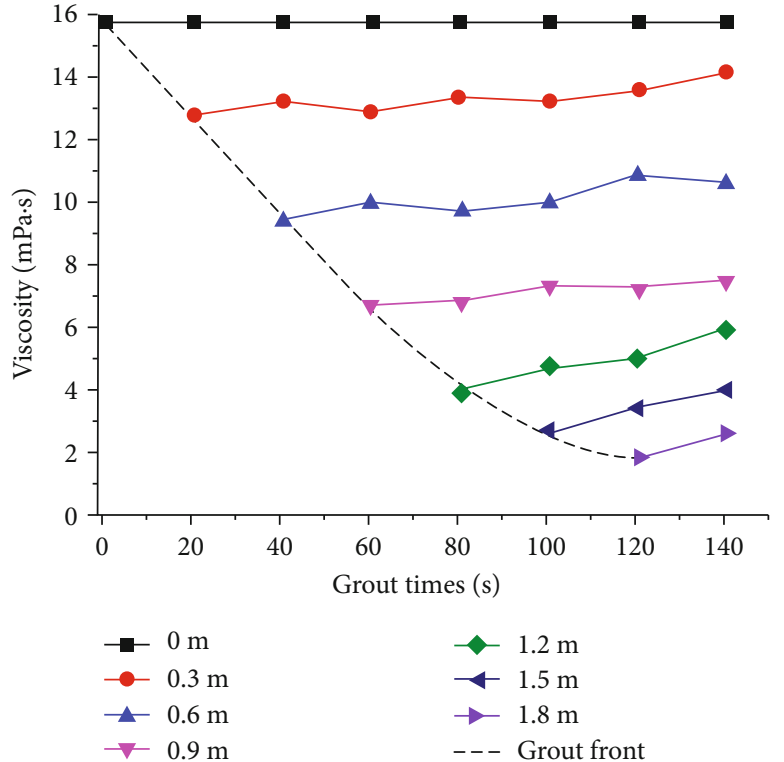

(a)

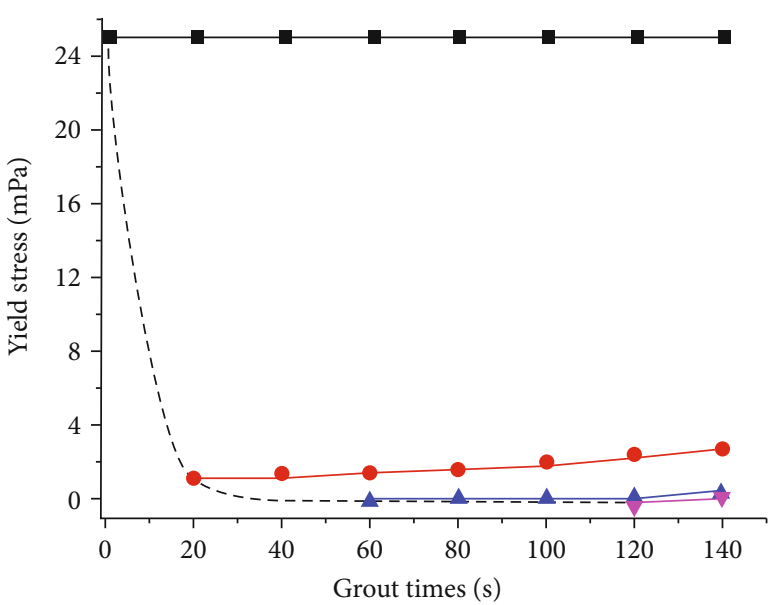

$\rightarrow-0 \mathrm{~m}$

$\longrightarrow 0.3 \mathrm{~m}$

$-0.6 \mathrm{~m}$

(b)

Figure 5: The temporal and spatial variation in slurry viscosity and yield stress in no.7 test ((a) the variation of slurry viscosity; (b) the variation of slurry yield stress).

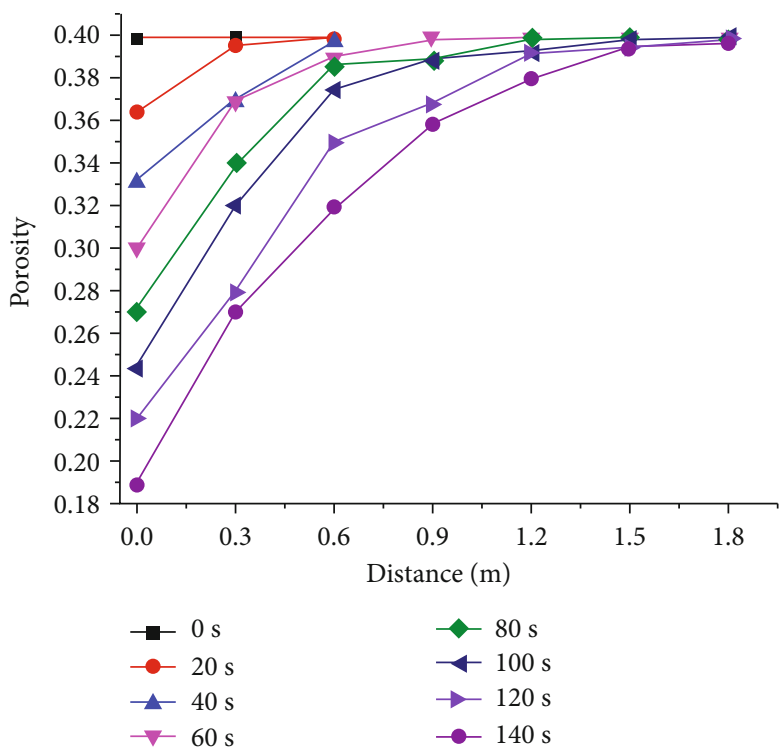

(a)

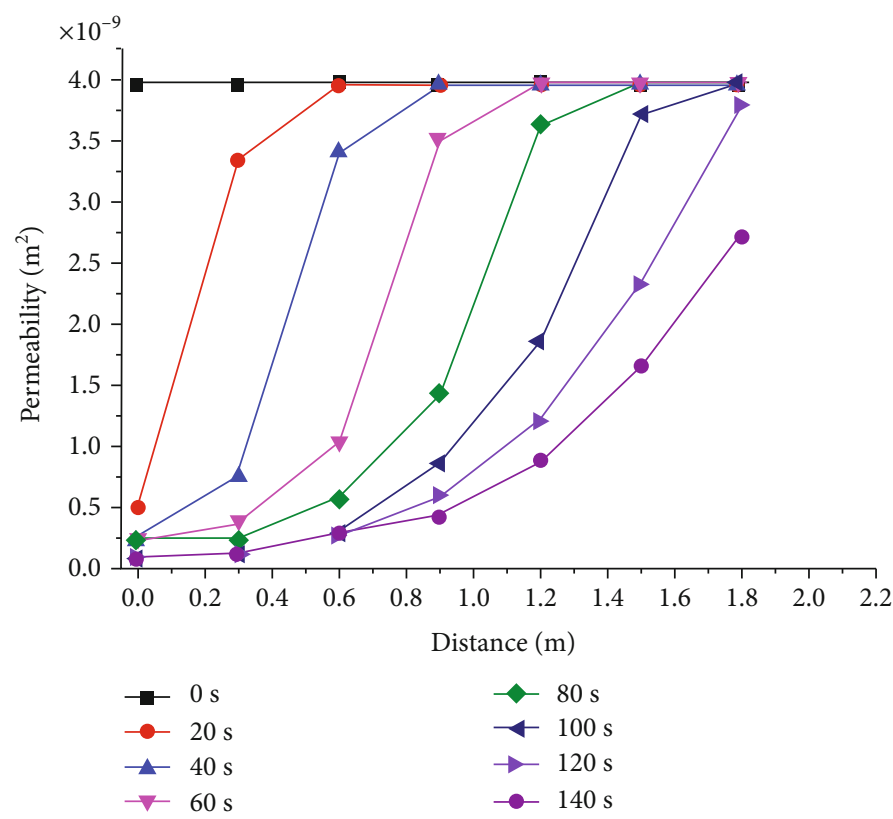

(b)

FIGURE 6: The temporal and spatial variation in porosity and permeability (a. the variation of porosity; b. the variation of permeability).

distance. As $-d p / d l$ is much larger than the starting pressure gradient $\zeta$, and equation (4) can be simplified as:

$$
\bar{v}=\frac{b^{2}}{8} \frac{1}{\mu}\left(-\frac{d p}{d l}-\frac{8}{3} \frac{\tau_{0}}{b}\right)
$$

The seepage velocity $v$, the average velocity of the single seepage pipeline $\bar{v}$, and the porosity of the injected medium $\varphi$ at any point in the porous media satisfy the following relation:

$$
v=\varphi \bar{v}
$$




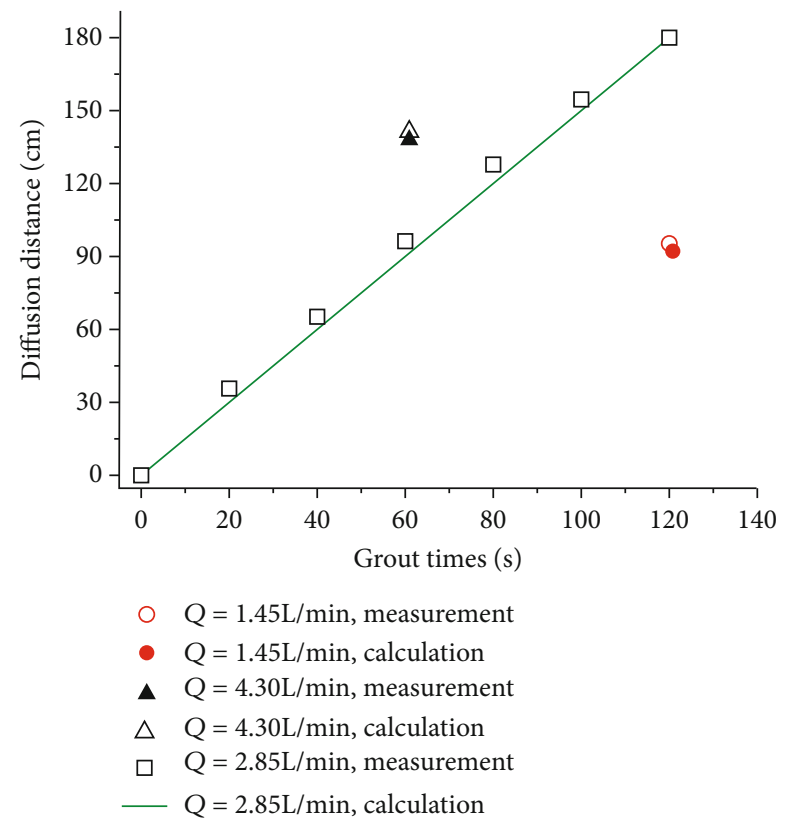

FIgUre 7: The grout diffusion distance with time.

The relation between permeability $k$ and the seepage channel radius $b$ is as follows:

$$
k=\frac{\varphi b^{2}}{8} .
$$

Under the condition of constant grout flow rate:

$$
Q=S v,
$$

where $Q$ is the total grouting rate and $S$ is the area of the slurry diffusion section.

According to equations (5)-(8), the pressure gradient in the slurry diffusion zone can be obtained by:

$$
\frac{d p}{d l}=-\frac{Q}{S k} \mu-\frac{2 \tau_{0}}{3} \sqrt{\frac{2 \varphi}{k}}, l<\frac{Q t}{\varphi S},
$$

where the slurry diffusion distance $l$ is equal to $Q t /(\varphi S)$ at any time.

3.3. Filtration. During the permeation grouting diffusion, cement-based particles were filtered by the porous media, which lead to flow paths being blocked and the stoppage of the flow [30-32]. Generally, lower cement particle content grouts have a lower risk of filtration. The filtration of cement-based grout occurs depending on many factors such as grout concentration, deposition time, and pore velocity, among others. Therefore, filtration leads to the temporal and spatial variation of the grout concentration and porosity, which have a significant influence on the permeation grouting diffusion.

The flow of cement-based grout through a porous medium can be analyzed by the advection-dispersion equation. According to previous research, the effect of hydrody- namic dispersion on the diffusion is small enough to ignore $[16,23]$. The governing equation is thus expressed as,

$$
\frac{\partial(\delta \varphi)}{\partial t}+\nabla \cdot(\nu \delta)=-\lambda \delta
$$

where $t$ is the deposition time, $\delta$ is the grout concentration, and $\lambda$ is the deposition coefficient with a dimension of $1 /$ (time).

A first-order rate law was used to describe the change of porosity of a porous medium [26].

$$
\frac{\partial \varphi}{\partial t}=\frac{\lambda c}{\rho_{s}}
$$

The key parameter in the continuum model was the deposition coefficient $\lambda$. This parameter is very difficult to estimate accurately. Generally, the values of $\lambda$ are regarded as a constant value $[16,29]$. However, after extensive parametric studies, Kim and Whittle [26] draw a conclusion that a constant value $a$ cannot describe the permeation grouting pressure in the column injection tests accurately. Hence, more complex modeling of filtration is necessary.

The determination of the deposition coefficient $\lambda$ is the key for a numerical model. A particle deposited in porous media could be described by the particle captured probability model. Because of the effect of ionic strength, $\mathrm{pH}$, and fluid velocity, the migrating particle in the porous media will be captured on the pore wall with a probability of $\theta$. A higher value $\theta$ suggests higher particle deposition potential. Rege and Fogler [33] postulated the $\theta$ value as an exponential function:

$$
\theta=\theta_{0} \cdot \exp \left(-\frac{v}{v_{\mathrm{cr}}}\right)
$$

where $\theta_{0}$ is a constant, depending on ionic conditions, $v$ is the pore flow velocity, and $v_{\mathrm{cr}}$ is the critical velocity where almost no particle deposits. In addition to the ionic strength, $\mathrm{pH}$, and fluid velocity, the particle size distribution could affect the deposition of cement particles significantly. An explicit expression of the deposition coefficient was adopted by Reddi and Bonala [34]. The expression uses a particle size distribution to calculate the particle accumulation in a soil filter. It is expressed as,

$$
\lambda=\frac{V}{a^{*} e^{2\left(f^{2}+m\right)}}\left[4(a \theta)^{2}-4(a \theta)^{3} e^{\left(f^{2}-2 m\right) / 2}+(a \theta)^{4} e^{2\left(f^{2}-2 m\right)}\right],
$$

where $a$ is the radius of migration particle, parameters $f$ and $m$ are the mean and standard deviation of $\log r, a^{*}$ is the effective pore length associated with each particle size distribution fraction. Actually, the pore distribution is directly related to the particle size distribution, if the shape of the particle is presumed to be spherical and the pore distribution is regarded as equivalent to bundles of cylinders. If the particle 


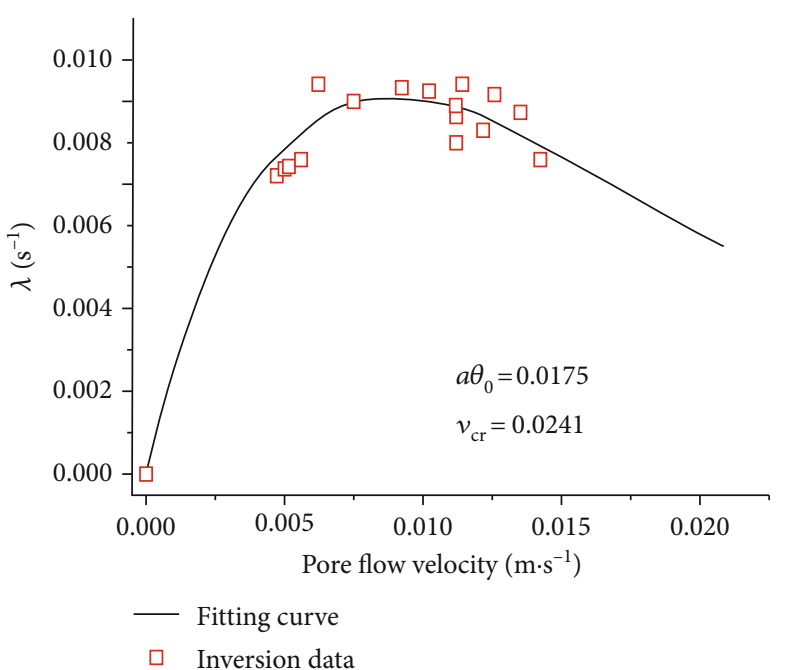

Figure 8: The nonlinear fitting of inversion data (WCR $=1.5, Q=$ $2.85 \mathrm{~L} / \mathrm{min})$.

size distribution is determined, the unknown parameters are the critical velocity $v_{\text {cr }}$ and $a \theta_{0}$.

The decrease of porous media porosity results in the decrease of permeability. The relationship between permeability and porosity can be described by the hyperbolic model $[16,35]$ :

$$
k=\frac{k_{0}}{1+\beta\left(\varphi-\varphi_{0}\right)},
$$

where $k_{0}$ is the initial permeability of the porous medium and $\beta$ is the damage coefficient.

3.4. Determination Method of Model Parameters. In the filtration model, the critical velocity $v_{c r}, a \theta_{0}$, and the damage coefficient $\beta$ are still unknown. The porosity and viscosity can be measured at the same position of the cylinder vessel. According to equation (3), the concentration of the grout is obtained. Then, the deposition coefficient can be calculated based on equation (11).

A one-dimensional permeation grouting experiment was carried out under a constant flow rate. The pore flow velocity $v$ was equal to the ratio of grout flow rate $Q$ to porosity $\varphi$. Then, the value of the deposition coefficient and the pore flow velocity can be used in the next fitting of the nonlinear curve.

In equation (12), the parameters $a^{*}, f$, and $m$ can be calculated based on the particle distribution of the sand. The inversion of the unknown parameters, the critical velocity $v_{\text {cr }}$ and $a \theta_{0}$, can be achieved by the fitting of the nonlinear curve. The results of the fitting are shown in Figure 8. The fitting was done with the software origin 9.0.

The permeability and porosity were measured in the permeation grouting experiments. As shown in Figure 9, the value of $\beta$ could be obtained by the fitting of the permeability and porosity. In this study, the value of $\beta$ fitted was 181.714 .

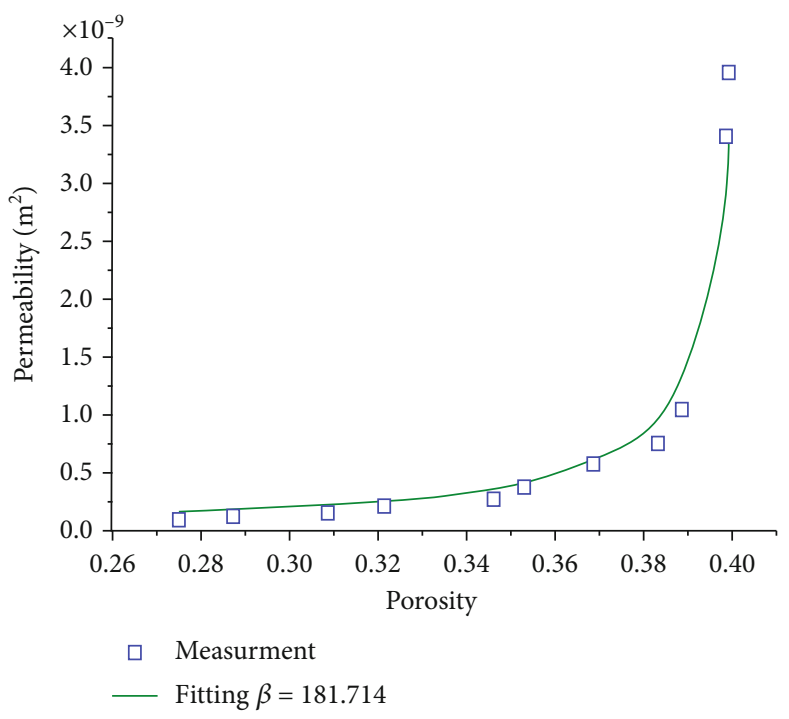

FIGURE 9: Relation of the permeability to the porosity.

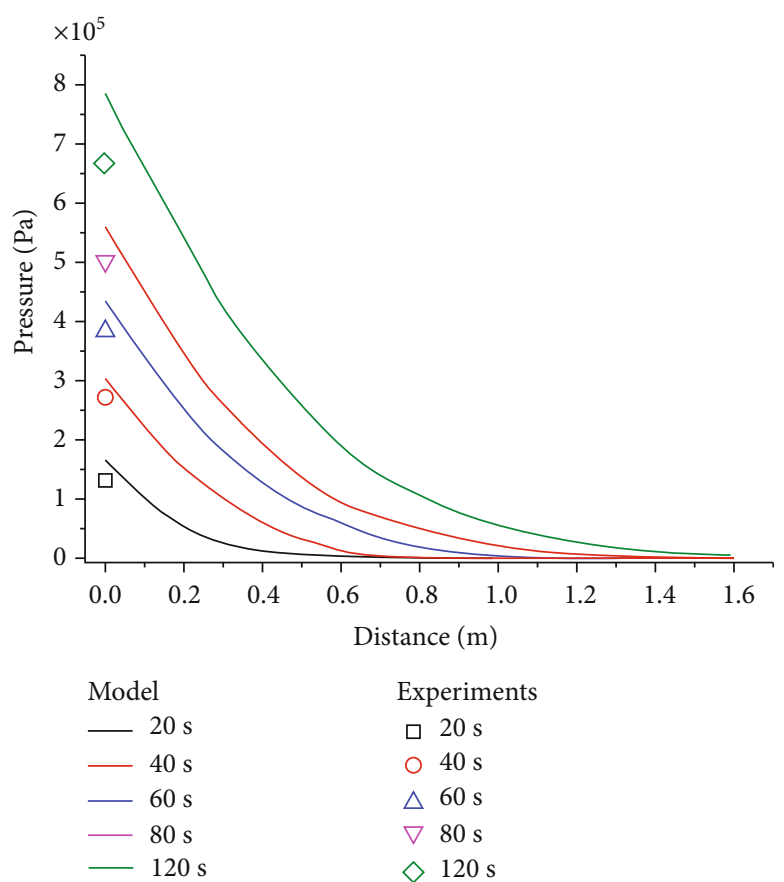

FIgURE 10: The temporal and spatial variation of grouting pressure $(\mathrm{Q}=2.85 \mathrm{~L} / \mathrm{min}$, WCR $=1.5)$.

\section{Verification of the Numerical Model}

In this section, the governing equations proposed were calculated using the finite element software COMSOL Multiphysics 5.1. The parameters used for the numerical calculations are as follows: initial porosity $n_{0}=0.40$, initial permeability $k_{0}=4.0 \times 10^{-9} \mathrm{~m}^{2}$, the density of cement particle $\rho_{\mathrm{s}}=2.92 \mathrm{~g} \cdot \mathrm{cm}^{-3}$, the density of water $\rho_{\mathrm{w}}=1.0 \mathrm{~g} \cdot \mathrm{cm}^{-3}, \beta=$ 181.714 , the critical velocity $v_{\mathrm{cr}}=0.0241 \mathrm{~m} \cdot \mathrm{s}^{-1}$, and $a \theta_{0}=$ 0.0175 . The numerical results were also compared with the experimental observations. 


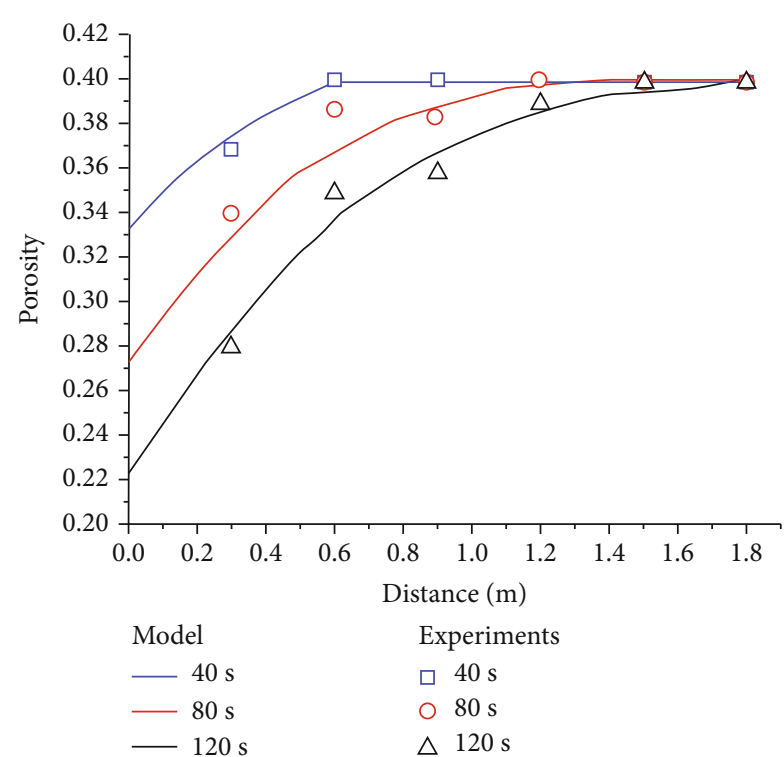

FIGURE 11: The spatial and temporal variation of porosity $(\mathrm{Q}=$ $2.85 \mathrm{~L} / \mathrm{min}$, $\mathrm{WCR}=1.5$ ).

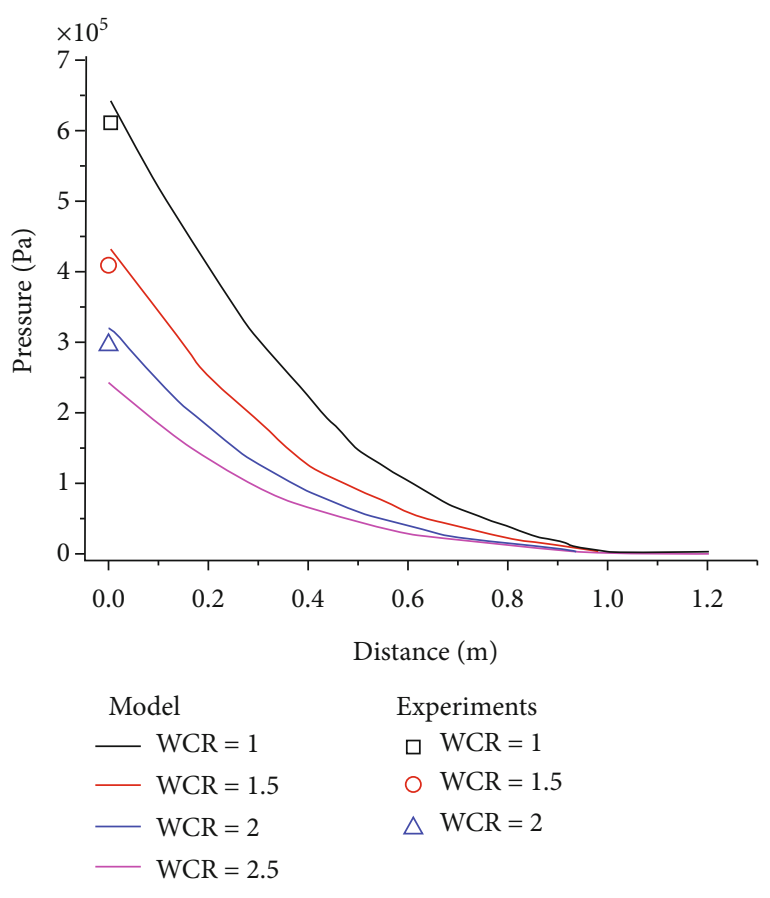

FIGURE 12: The variation of grouting pressure versus the diffusion distance $(Q=2.85 \mathrm{~L} / \mathrm{min}, t=60 \mathrm{~s})$.

As shown in Figure 10, the grouting pressure decreases dramatically along the grout diffusion distance. Compared to the measurement, the numerical model could capture the grouting pressure at low grouting pressure. The equations overestimated the grouting pressure. Yoon and El Mohtar [36] reported that the ionic strength decreases with the increase in grouting pressure because a high pressure may fracture the skeleton and increase the number and apertures of the flow channels, thereby reducing the probability of filtration. However, in the numerical model, the $\theta_{0}$ was

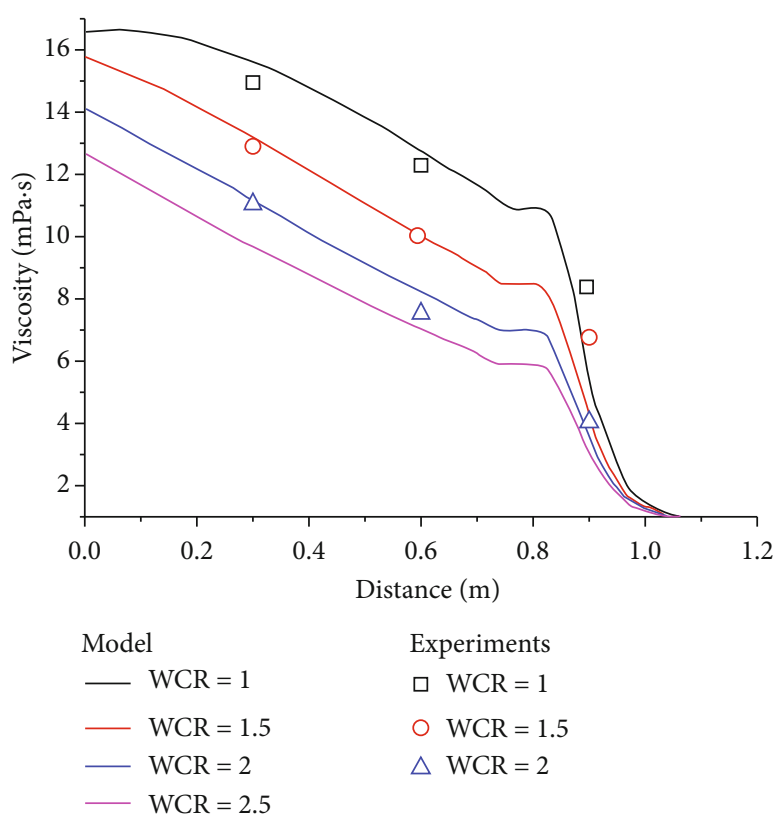

FIgURE 13: The variation of grout viscosity versus the diffusion distance $(Q=2.85 \mathrm{~L} / \mathrm{min}, t=60 \mathrm{~s})$.

treated as a constant value, which depended on ionic conditions. Therefore, the grouting pressure measurement was lower than that from the calculation. From Figure 11, the porosity calculated was in good agreement with the measurements. Because the parameters used in the numerical model were inverted by the porosity and other measurements, the effect of the grouting pressure on the ionic strength may be covered up.

\section{Discussion}

Particle size distribution parameters and grouting parameters play a key role in the grout diffusion in porous media. The effect of suspension particle size distribution on the diffusion has been discussed by Reddi et al. [34]. In this research, the effect of the water/cement ratio and the grout flow velocity are studied.

5.1. The Influence of Water/Cement Ratio. Figure 12 presents the spatial and temporal variation of grouting pressure versus the grout diffusion distance. As shown in the figure, when the diffusion distance is less than $1.2 \mathrm{~m}$, the smaller the watercement ratio is, the more obvious the nonlinear attenuation of the slurry pressure along the diffusion distance is. At the same location, the results of experiments with different water/cement ratios show that the grouting pressure tends to be lower with a higher water/cement ratio. Furthermore, the grouting pressure measurements fit the calculation well. In Figure 13, the reduction of grout viscosity along the diffusion distance is linear, approximately. Moreover, the reduction rate with different water/cement ratio is similar.

5.2. Grout Flow Velocity. The prominent impact of the flow velocity on the filtration effect can be seen in Figure 14. The 


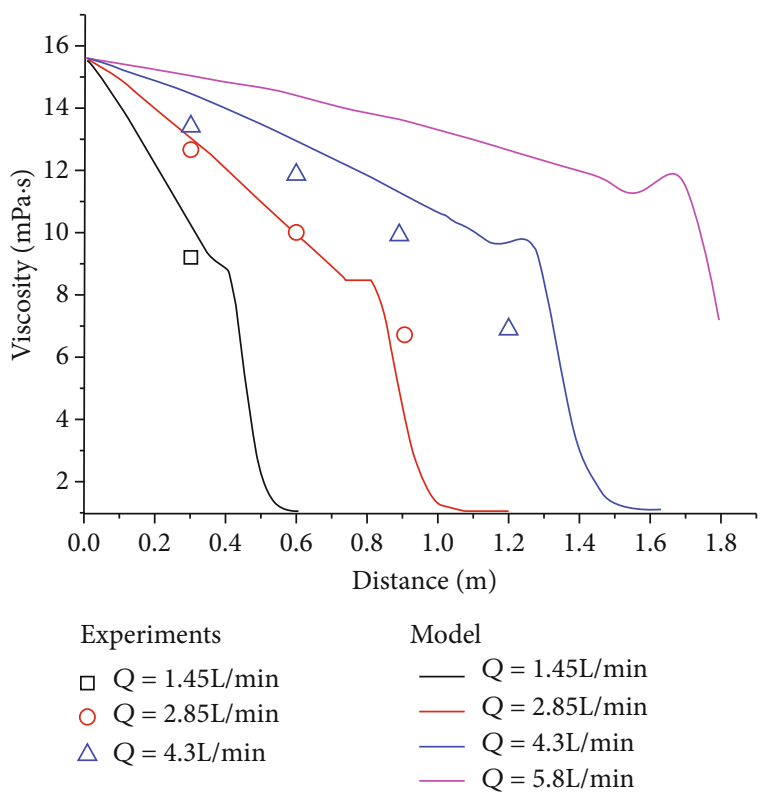

FIGURE 14: Variation in the viscosity of the effluent versus the distance under different flow rates (WCR $=1.5, t=60 \mathrm{~s}$ ).

four groups of experiments with different grouting flow velocities show that the grout viscosity tends to be lower under the condition of a lower grouting flow rate. A lower viscosity indicates a stronger filtration effect. In the process of permeation grouting, there is a critical velocity for cement particles to deposit in the porous media. When the migration velocity of cement particles reaches the critical velocity, the deposition velocity of cement particles reaches its maximum. Generally, the critical velocity in permeation grouting is smaller than the grout flow velocity in actual permeation projects. In addition, the grout pore velocity and the porosity vary with time due to the filtration. For these reasons, critical velocity is often difficult to observe. Although a higher grout flow velocity can benefit from the grout diffusion, a higher flow velocity will lead to a higher grouting pressure, which will increase the potential failure of the soil structure.

\section{Conclusion}

The main features of the diffusion of cement-based grouts in porous media were investigated through a series of laboratory tests under the constant flow velocity condition. Based on the results of experiments, a numerical model was established to describe the cemented gout permeation diffusion. The main conclusions from the experimental and numerical results are summarized as follows:

(1) One-dimensional permeation grouting test was carried out under the constant flow velocity condition. There were dramatic variations in rheological parameters and porosity along the diffusion distance. However, the rheological parameters changed slightly with time in the same position. The parameters in the model were determined based on the test results
(2) By introducing the time-space variation equation for porosity and permeability of porous media considering the filtration effect, a numerical model considering the filtration effect and grout rheological properties was established. Furthermore, the rationality of the theoretical calculation model was verified by the results of the experiments

(3) The effect of the water/cement ratio and the grout flow velocity were studied. The filtration coefficient was found to rapidly decrease with the increase of pore velocity. The grouts with different water/cement ratio show similar filtration behavior in the permeation grouting diffusion

\section{Data Availability}

The data used to support the findings of this study are available from the corresponding author upon request.

\section{Conflicts of Interest}

The authors declare that there is no conflict of interest regarding the publication of this paper.

\section{Acknowledgments}

This research was financially supported by the National Natural Science Foundation of China (Grant Nos.U1706223, 51808359, 51908329, and 52009075), the Natural Science Foundation of Shandong Province (No. ZR2018MEE047), and Hebei Provincial Natural Science Foundation of China (E2019210356).

\section{References}

[1] D. Gouvenot, "State of the art in European grouting," Proceedings of the Institution of Civil Engineers-Ground Improvement, vol. 2, no. 2, pp. 51-67, 1998.

[2] H. Wang, Q. Liu, S. Sun, Q. Zhang, Z. Li, and P. Zhang, "Damage model and experimental study of a sand groutingreinforced body in a seawater environment," Water, vol. 12, no. 9, p. 2495, 2020.

[3] X. Du, H. Fang, S. Wang, B. Xue, and F. Wang, "Experimental and practical investigation of the sealing efficiency of cement grouting in tortuous fractures with flowing water," Tunnelling and Underground Space Technology, vol. 108, no. article 103693, 2020.

[4] Z. L. Zhou, X. M. Du, Z. Chen, and Y. L. Zhao, "Grouting diffusion of chemical fluid flow in soil with fractal characteristics," Journal of Central South University, vol. 24, no. 5, pp. 1190-1196, 2017.

[5] Z. Zhou, X. Du, S. Wang, X. Cai, and L. Chen, "Micromechanism of the diffusion of cement-based grouts in porous media under two hydraulic operating conditions: constant flow rate and constant pressure," Acta Geotechnica, vol. 14, no. 3, pp. 825-841, 2019.

[6] J. H. Yin, L. J. Su, R. W. M. Cheung, Y. K. Shiu, and C. Tang, "The influence of grouting pressure on the pullout resistance of soil nails in compacted completely decomposed granite fill," Geotechnique, vol. 59, no. 2, pp. 103-113, 2009. 
[7] Z. Zhou, H. Zang, S. Wang, X. Du, D. Ma, and J. Zhang, "Filtration behaviour of cement-based grout in porous media," Transport in Porous Media, vol. 125, no. 3, pp. 435-463, 2018.

[8] N. D. Ahfir, H. Q. Wang, A. Benamar, A. Alem, N. Massei, and J. P. Dupont, "Transport and deposition of suspended particles in saturated porous media: hydrodynamic effect," Hydrogeology Journal, vol. 15, no. 4, pp. 659-668, 2007.

[9] F. Compère, G. Porel, and F. Delay, “Transport and retention of clay particles in saturated porous media. Influence of ionic strength and pore velocity," Journal of Contaminant Hydrology, vol. 49, no. 1-2, pp. 1-21, 2001.

[10] L. Bennacer, N. D. Ahfir, A. Bouanani, A. Alem, and H. Q. Wang, "Suspended particles transport and deposition in saturated granular porous medium: particle size effects," Transport in Porous Media, vol. 100, no. 3, pp. 377-392, 2013.

[11] A. Alem, N. D. Ahfir, A. Elkawafi, and H. Wang, "Hydraulic operating conditions and particle concentration effects on physical clogging of a porous medium," Transport in Porous Media, vol. 106, no. 2, pp. 303-321, 2015.

[12] N. D. Ahfir, A. Benamar, A. Alem, and H. Wang, "Influence of internal structure and medium length on transport and deposition of suspended particles: a laboratory study," Transport in Porous Media, vol. 76, no. 2, pp. 289-307, 2009.

[13] M. Zaheer, Z. Wen, H. B. Zhan, X. L. Chen, and M. G. Jin, “An experimental study on solute transport in one dimensional clay soil columns," Geofluids, vol. 2017, 17 pages, 2017.

[14] O. S. Q. Yousif, M. Karakouzian, N. O. A. Rahim, and K. A. Rashed, "Physical clogging of uniformly graded porous media under constant flow rates," Transport in porous media, vol. 120, no. 2, pp. 1-17, 2017.

[15] O. Chupin, N. Saiyouri, and P. Y. Hicher, "The effects of filtration on the injection of cement-based grouts in sand columns," Transport in Porous Media, vol. 72, no. 2, pp. 227-240, 2008.

[16] Z. Saada, J. Canou, L. Dormieux, J. C. Dupla, and S. Maghous, "Modelling of cement suspension flow in granular porous media," International Journal for Numerical and Analytical Methods in Geomechanics, vol. 29, no. 7, pp. 691-711, 2005.

[17] M. Auset and A. A. Keller, "Pore-scale visualization of colloid straining and filtration in saturated porous media using micromodels," Water resources research, vol. 42, no. 12, 2006.

[18] G. Seidler, G. Martinez, L. Seeley et al., "Granule-by-granule reconstruction of a sandpile from x-ray microtomography data," Physical Review E, vol. 62, no. 6, article 8175, 2000.

[19] D. Wildenschild, C. Vaz, M. Rivers, D. Rikard, and B. Christensen, "Using X-ray computed tomography in hydrology: systems, resolutions, and limitations," Journal of Hydrology, vol. 267, no. 3-4, pp. 285-297, 2002.

[20] Y. Kutsovsky, L. Scriven, H. Davis, and B. Hammer, "NMR imaging of velocity profiles and velocity distributions in bead packs," Physics of Fluids, vol. 8, no. 4, pp. 863-871, 1996.

[21] E. Fukushima, "Nuclear magnetic resonance as a tool to study flow," Annual Review of Fluid Mechanics, vol. 31, no. 1, pp. 95123, 1999.

[22] L. N. Reddi, X. Ming, M. G. Hajra, and I. M. Lee, "Permeability reduction of soil filters due to physical clogging," Journal of Geotechnical and Geoenvironmental Engineering, vol. 126, no. 3, pp. 236-246, 2000.

[23] J. S. Kim, I. M. Lee, J. H. Jang, and H. Choi, "Groutability of cement-based grout with consideration of viscosity and filtration phenomenon," International Journal for Numerical and
Analytical Methods in Geomechanics, vol. 33, no. 16, pp. 1771-1797, 2009.

[24] D. C. Mays and J. R. Hunt, "Hydrodynamic aspects of particle clogging in porous media," Environmental Science and Technology, vol. 39, no. 2, pp. 577-584, 2005.

[25] A. Alem, A. Elkawafi, N. D. Ahfir, and H. Wang, "Filtration of kaolinite particles in a saturated porous medium: hydrodynamic effects," Hydrogeology Journal, vol. 21, no. 3, pp. 573586, 2013.

[26] Y. S. Kim and A. J. Whittle, "Particle network model for simulating the filtration of a microfine cement grout in sand," Journal of Geotechnical and Geoenvironmental Engineering, vol. 135, no. 2, pp. 224-236, 2009.

[27] D. Ma, M. Rezania, H. S. Yu, and H. B. Bai, "Variations of hydraulic properties of granular sandstones during water inrush: effect of small particle migration," Engineering Geology, vol. 217, pp. 61-70, 2017.

[28] K. K. Sio, Properties of Cement Based Permeation Grout Used in Ground Engineering, Doctoral dissertation, 2006.

[29] F. Bouchelaghem and L. Vulliet, "Mathematical and numerical filtration-advection-dispersion model of miscible grout propagation in saturated porous media," International Journal for Numerical and Analytical Methods in Geomechanics, vol. 25, no. 12, pp. 1195-1227, 2001.

[30] J. P. Herzig, D. M. Leclerc, and P. L. Goff, "Flow of suspensions through porous media-application to deep filtration," Industrial \& Engineering Chemistry, vol. 62, no. 5, pp. 8-35, 1970.

[31] G. Gustafson and H. Stille, "Prediction of groutability from grout properties and hydrogeological data," Tunnelling and Underground Space Technology, vol. 11, no. 3, pp. 325-332, 1996.

[32] M. Axelsson, G. Gustafson, and A. Fransson, "Stop mechanism for cementitious grouts at different water-to-cement ratios," Tunnelling and Underground Space Technology, vol. 24, no. 4, pp. 390-397, 2009.

[33] S. D. Rege and H. S. Fogler, "A network model for deep bed filtration of solid particles and emulsion drops," AICHE Journal, vol. 34, no. 11, pp. 1761-1772, 1998.

[34] L. N. Reddi and M. V. S. Bonala, "Analytical solution for fine particle accumulation in soil filters," Journal of Geotechnical and Geoenvironmental Engineering, vol. 123, no. 12, pp. 11431152, 1997.

[35] T. Iwasaki, J. J. Slade, and W. E. Stanley, "Some notes on sand filtration," Journal (American Water Works Association), vol. 29, no. 10, pp. 1591-1597, 1937.

[36] J. Yoon and C. S. El Mohtar, "Groutability of granular soils using bentonite grout based on filtration model," Transport in Porous Media, vol. 102, no. 3, pp. 365-385, 2014. 